\title{
PER RECTAL MAGNETIC INFRA- RED LASER THERAPY BY OPTICAL PROBE AND ITS EFFECT ON PELVIC PAIN, DYSURIAAND HAEMOSPERMIA OF THE PATIENTS OF CHRONIC PELVIC PAIN SYNDROME
}

\author{
A.M. ANAMUR RASHID CHOUDHURY ${ }^{1}$, MD. WALIUL ISLAM ${ }^{1}$, TASMINA PARVEEN ${ }^{2}$, \\ PARVEEN SULTANA ${ }^{3}$
}

${ }^{1}$ Department of Urology, National Institute of Kidney Diseases and Urology.Dhaka, ${ }^{2}$ Department of Physiology, Uttara Adhunik Medical College, Uttara, Dhaka, ${ }^{3}$ Department of Forensic Medicine, Enam Medical College Hospital.

\begin{abstract}
:
Objective : To observe the improvement of the symptoms of pelvic pain, dysuria and haemospermia after treatment with magnetic infrared laser therapy in patients with chronic pelvic pain syndrome.

Methods : This study was an observational study done on 30 male patients with pelvic pain and age range varied from 30 to 60 years. The study was conducted from 2009 to 2011 in a private medical center in Dhaka city. The MIL-therapy in use has a wave length of $904 \mathrm{~nm}$ and a frequency of $3000 \mathrm{~Hz}$. The Laser beam reaches the prostate with a special optic probe. In this study the patients were examined per rectally by doing digital rectal examination with use of gloves and gel. At the beginning we used a "Laser Super Sonic" machine with endorectal probe according to Strada. The treatment schedule was one treatment every two days (treatment's time of 5/10 minutes, wave length $1000 \mathrm{KHz}$ )) for a total of 10 applications. After 10 applications patients were asked for follow-up and data were callected and analysed.
\end{abstract}

Results : Out of 30 patients of chronic pelvic pain syndrome aged between 30-60 yrs with compains of pelvic pain, dysuria and haemospermia were under treatment with MIL therapy. 18 patients with pelvic pain, 13 patients with dysuria and 04 patients with haemospermia were cured after laser treatment. There were improvement of symptoms of pelvic pain in 11 patients, dysuria in 07 patients after laser therapy. But 01 patient found not improved . Among 30 cases 23 patients found normal sized prostate and 07 found enlarged prostate on digital rectal examination and on USG findings, 21 were found normal sized, 09 found enlarged sized prostate and 07 patients had increased PVR .

Conclusion : Patient sign and symptoms improved significantly after $M / L$ therapy.

Key words: MIL Therapy, Chronic pelvic pain syndrome (CPPS).

Bangladesh J. Urol. 2014; 17(1): 42-46

\section{Introduction :}

Laser Therapy is a regulative medical treatment modality, which is used most in medical specialties including other than urological aspect such as Dermatology, Traumatology, Sports Medicine, Orthopedics, Dental Medicine, Gynecology, General Medicine etc. ${ }^{1}$ Laser means Light Amplification by Stimulated Emission of

Correspondence : A.M. Anamur Rashid Choudhury, Department of Urology, National Institute of Kidney Diseases and Urology. Dhaka. E-mail: dr_choudhury2001@yahoo.com
Radiation and was first theorized by Einstein. In 1960 Miaman developed the first laser, a ruby laser. This was a tube laser with a metal chamber, which contained the element ruby. When an electrical current excites the enclosed element, the atoms give off photons or packets of light energy. ${ }^{1}$ MIL therapy is based on a combinative mutually reinforcing influence of impulse infrared laser irradiation, pulsating red light, pulsating wide-band infrared irradiation and static magnetic field on a biological object .Laser is a technical device, which 
emits in the form of a focused directive beam a coherent monochromatic polarized electromagnetic irradiation, i.e. the light in a very narrow spectral region. Chronic prostatitis or chronic pelvic pain syndrome (CP/CPPS) occurs in men and is characterized by persistent discomfort or pain in the pelvic area that lasts several months, often longer. The discomfort is usually at the base of the penis and around the anus and lower back. Sometimes it spreads into the testes. Some patients have pain with ejaculation, and others may have pain or an urgency or hesitancy when they pass urine, as well as a poor urinary stream. The cause of CP/CPPS is not known. ${ }^{2,3}$ Prostatitis is a disease entity that is diagnosed by symptoms, microscopy of expressed prostatic secretions (EPS) and culture of EPS and segmented urine samples. It is a common medical condition. ${ }^{4,5}$

Prostatitis is any form of inflammation of the prostate gland. Because women do not have a prostate gland, it is a condition only found in men, although women do have microscopic paraurethral Skene's glands connected to the distal third of the urethra in the prevaginal space that are homologous to the prostate, and may cause symptoms. ${ }^{6}$ According to the 1999 National Institute of Health (NIH) Classification, there are four categories of prostatitis: 7,8

Category I : Acute prostatitis (bacterial)

Category II : Chronic bacterial prostatitis

Category III : Chronic prostatitis/chronic pelvic pain syndrome (CP/CPPS), Pelvic MyoneuropathySubdivisions of IIIa (inflammatory) and IIIb (noninflammatory) exist based on levels of pus cells in expressed prostatic secretions, but these subcategories are of limited use clinically

Category IV : Asymptomatic inflammatory prostatitis In chronic prostatitis/chronic pelvic pain syndrome (CPI CPPS) there is

- Pelvic pain of unknown cause, lasting longer than 6 months, as the key symptom. Symptoms may wax and wane. Pain can range from mild discomfort to debilitating. Pain may radiate to back and rectum, making sitting difficult.

- Dysuria, arthralgia, myalgia,

- Unexplained fatigue,

- Abdominal pain, and frequency may all be present.
- Frequent urination and increased urgency may suggest interstitial cystitis (inflammation centred in bladder rather than prostate).

- Ejaculation may be painful, as the prostate contracts during emission of semen, although nerveand muscle-mediated post-ejaculatory pain is more common, and a classic sign of CP/CPPS.

- Passage of blood with semen during ejaculation

- Some patients report low libido, sexual dysfunction and erectile difficulties. Pain after ejaculation is a very specific complaint that distinguishes CP/CPPS from men with BPH or normal men.

There are no definitive diagnostic tests for CP/CPPS. This is a poorly understood disorder, even though it accounts for $90 \%-95 \%$ of prostatitis diagnoses. It is found in men of any age, with the peak onset in the early 30s. CP/CPPS may be inflammatory (category IIIa) or non-inflammatory (category IIIb). In the inflammatory form, urine, semen, and other fluids from the prostate contain pus cells (dead white blood cells or WBCs), whereas in the non-inflammatory form no pus cells are present. Recent studies have questioned the distinction between categories IIIa and IIIb, since both categories show evidence of inflammation if pus cells are ignored and other more subtle signs of inflammation, like cytokines, are measured. Normal men have slightly more bacteria in their semen than men with chronic prostatitis/pelvic myoneuropathy. Men with CP/CPPS are more likely than the general population to suffer from Chronic Fatigue Syndrome (CFS), and Irritable Bowel Syndrome (IBS). Prostate specific antigen levels may be elevated, although there is no malignancy. ${ }^{5,6,10}$

Laser therapy has analgesic effect due to Measurable changes in the potential of nerve cell membranes lead to its hyperpolarization as a good analgesic mechanism. It has improves release reaction of endorphines and there is changes in transmitter concentration at the synapeis.

Laser therapy also except anti - Inflammatory effect because itn enhances proliferation of immune cells (enhancement of immune response, increase of NK cell activity etc.), Enhances lymphatic activity (drainage) and Improves microcirculation (vasodilation)

Over all pathophysiology of laser therapy in the correct frequency, the red light is absorbed within the mitochondria and the infrared light is absorbed at the cell membrane. In the cell of prostate gland this results in a change in membrane permeability, increased ATP levels and increased DNA production. So there is cellular 
hyperplasia of prostate and increased activity of ATP dependant Na-K pump ${ }^{1,2,3}$.

\section{Materials and methods:}

This study was an observational study done on 30 male patients with pelvic pain and age range varied from 30 to 60 years. The study period was from 2009 to 2011 in a private medical center at Dhaka city. The MIL-therapy in use has a wave length of $904 \mathrm{~nm}$ and a frequency of $3000 \mathrm{~Hz}$. The Laser beam reaches the prostate with a special optic probe. In this study the patient is examined per rectally after doing digital rectal examination with use of gloves and gel. It can be sterilized and it is atoxic. At the beginning we used a "Laser Super Sonic" machine with endorectal probe according to Strada. The treatment schedule was one treatment every two days (treatment's time of $5 / 10$ minutes, wave length $1000 \mathrm{KHz}$ )) for a total of 10 applications. Transrectal laser therapy was not indicated in prostate larger than $4 \mathrm{~cm}$ because this is the maximum depth of the laser beam's efficacy.

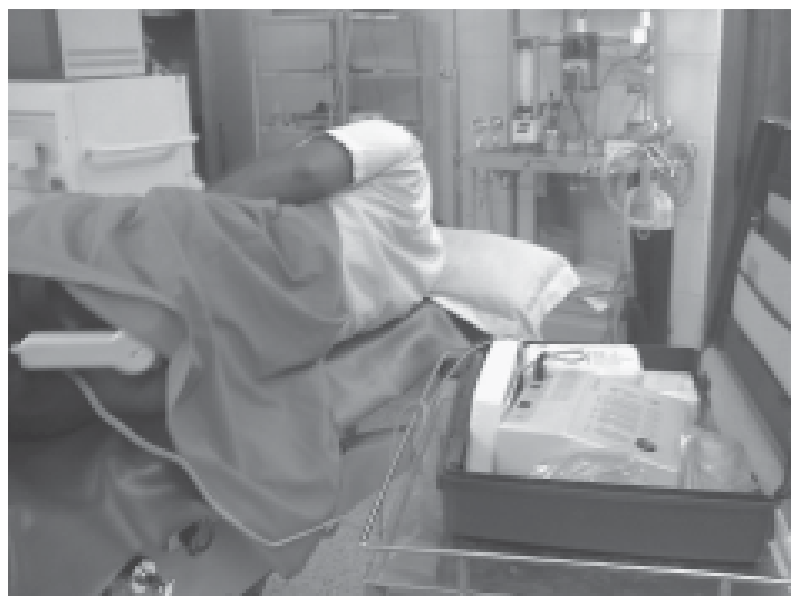

Fig I: MIL instrument

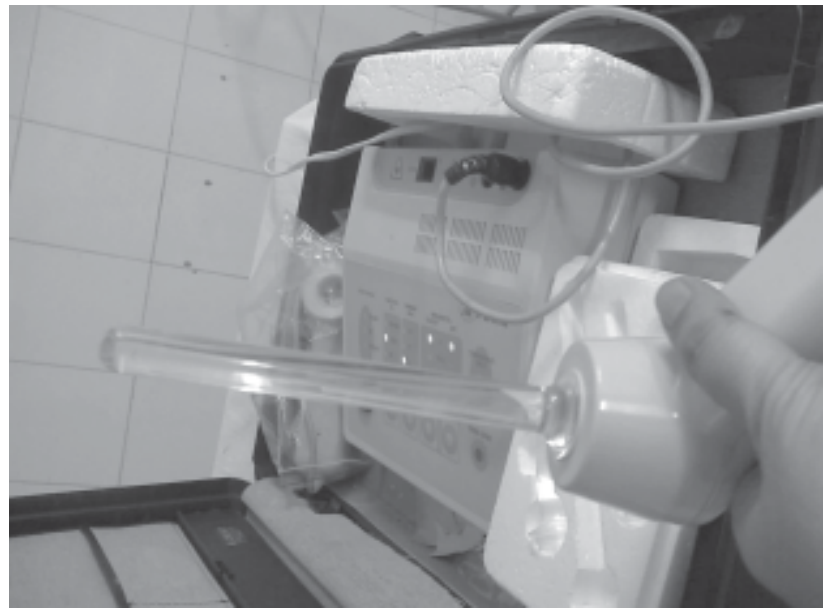

Fig.-2: Optical Probe

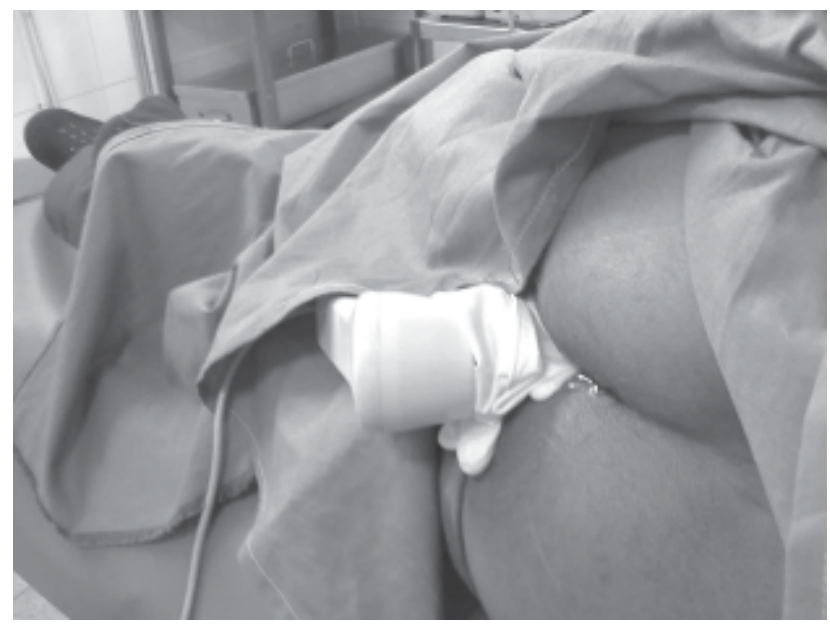

Fig.3:Per rectally laser therapy by optical probe

\section{Results :}

Out of 30 cases studied, age of the patients was between 30 to 60 years. Table-I shows age distribution of the patients.

Table-I

Distribution of patients by age $(n=30)$

\begin{tabular}{lcc}
\hline $\begin{array}{l}\text { Age of the patients) } \\
\text { (yrs }\end{array}$ & $\begin{array}{c}\text { Number of } \\
\text { patients }(\mathrm{n})\end{array}$ & $\begin{array}{c}\text { Percentage } \\
(\%)\end{array}$ \\
\hline $31-40$ & 13 & 43 \\
$41-50$ & 8 & 27 \\
$51-60$ & 9 & 30 \\
\hline
\end{tabular}

Table-II

Distribution of patients by symptomatology $(n=30)$

\begin{tabular}{lcc}
\hline Symptoms & Number of patients & Percentage \\
\hline Pelvic pain & 30 & 100 \\
Dysuria & 20 & 67 \\
Haemospermia & 4 & 13 \\
\hline
\end{tabular}

Among 30 patients all patients had pelvic pain and 20 had dysuria, only 04 patients had haemospermia.

Table-III

Digital rectal examination (DRE) finding of the study $\operatorname{cases}(n=30)$

\begin{tabular}{lcc}
\hline DRE finding & Number of patients & Percentage \\
\hline Enlarged prostate & 7 & 23 \\
Normal sized prostate & 23 & 77 \\
Any other finding & 0 & 0 \\
\hline
\end{tabular}

After DRE, among 30, 23 patients found normal sized prostate and only 07 patients found enlarged prostate. 
Table-IV

Ultrasonogram (USG) finding of the patients(n)

\begin{tabular}{lcc}
\hline USG finding & Number of patients & Percentage \\
\hline Increased PVR & 7 & 23 \\
Enlarged prostate & 9 & 30 \\
Normal sized prostate & 21 & 70 \\
Any other & 0 & 0 \\
\hline
\end{tabular}

Table IV showing USG findings and found 21 patients had normal sized prostate and only 09 patients had enlarged prostate .07 patients found increased PVR

Table-V

Improvement of symptoms after laser treatment

\begin{tabular}{lccc}
\hline Symptoms & Cured & Improved & Not improved \\
\hline Pelvic pain & 18 & 11 & 1 \\
Dysuria & 13 & 7 & 0 \\
Haemospermia & 4 & 0 & 0 \\
\hline
\end{tabular}

After per rectal laser therapy by optical probe there were improvement of symptoms found. Patients with pelvic pain, about 18 were cured, 11 were improved and only 01 was not improved. Patients with dysuria, about 13 were cured, 07 were improved. Patients with haemospermia, all (04) patients were cured.

Table-VIII

Complications after laser treatment $(n=30)$

\begin{tabular}{lcc}
\hline Significant complication & $\begin{array}{c}\text { Number of } \\
\text { patients }\end{array}$ & Percentage \\
\hline Yes & 0 & 0 \\
No & 30 & 0 \\
\hline
\end{tabular}

There were no significant complication found after per rectal laser treatment.

\section{Discussion:}

Chronic prostatitis/Chronic pelvic pain syndrome(CPPS) is a very uncomfortable condition affecting males from very young age to above 70 s. The exact clean cut diagnosis is very difficult. Many patients of such condition end up with unnecessary protatectomies and bladder neck incisions (BNI), having no effective recovery. This made the scientists and urologists desire to find out ways of recovery from this benign but devastating situation. Magnetic Infra Red Laser therapy(MIL-therapy) is a possible answer to alleviate this problem. This technique has not gained much popularity in our country as yet. Awareness of its effect having minor or no complication and further study are needed to gain its acceptance by clinicians.

Literature demonstrates, CPPS may occur from adolescence to old age.11 But this study shows youngest presentation at the age of 31 years. This may be due to the fact that our social infrastructure creates a feeling of shyness among the adolescent population to seek medical advice for such problems. The oldest patient in this series is 60 years. This could be due to lower life expectancy of people of this area.

Considering the symptomatology, pelvic pain and discomfort are the most common presentation which were present in $100 \%$ of the case studied. This correlates exactly with other different studies[12,13,14]. Other presentations include dysuria, haemospermia. The findings together with their frequency are exactly similar to certain other studies[12,13,14].

Digital rectal examination (DRE) is necessary mainly to exclude LUTS due to prostatic enlargement- benign or malignant14. Prostate size was normal in $77 \%$ cases and mild enlargement in $23 \%$ cases.

Post void residual volume(PVR) and prostate size were found to be normal in most of the cases in this series. Literature also illustrates the same findings[13,15].

After completion of laser therapy, patients were assessed for their improvement and they were followed up for a period of six months. Improvement of symptoms were graded on a subjective scale of cured, improved and not improved. As the scoring is subjective, there is an element of fallacy. It was found that, there was hardly any patient who did not improve. Majority were cured and rest had improvement with only one patient having no improvement of any symptom. Studies in Europe and America describes around $100 \%$ recovery after laser therapy[$[16,17,18]$. The magic bullet is, there is no gross or significant complication following laser treatment both in literature and in this study[18].

\section{Conclusion:}

Magnetic infra red laser therapy (MIL-therapy) gives a fruitful effect on chronic pelvic pain syndrome (CPPS). The following short article gives a very good summary of the benefits of laser therapy. CPPS, a painful type of Chronic Prostatitis frequently troubles man for years. Among many sign and symptoms of CPPS, pelvic pain, dysuria and haemospermia were observed and found very good effect after laser therapy . In this study it was proved that improvement of pain occur after applying MIL- therapy.

Conflict of Interest : None Declared 


\section{References :}

1. (http://wwww.finetreatment.co.uk/natural-alternative/ prostate-treatment.html)

2. Nickel JC. Pathogenesis of Chronic Protatitis. $\mathrm{Br}$ J Urol; 1990; 66: 47-49.

3. R.B. Alexander, K.J. Propert, A.J. Schaeffer, J.R. Landis, J.C. Nickel, M.P. O'Leary et al; Treating Men with Chronic Prostatitis/Chronic Pelvic Pain Syndrome, Annals of Internal Medicine; 2004, 141: 581-589.

4. Litwin MS. The National Institute of Health Chronic Prostatitis Symptom Index: development and validation of a new outcome measure. Chronic Prostatitis Collaborative Research Network. J Urol 1999; 162: 369-375.

5. Nickel JC, Nyberg LM, Hennenfent M. Research guidelines for chronic prostatitis: consensus report from the first National Institutes of Health International Prostatitis Collaborative Network. Urology 1999; 54: 229-233. .

6. Ammerican top urologists Wikipedia

7. Kaplan SA. Transurethral microwave hyperthermia and transurethral needle ablation. Prostate Cancer and Prostatic Diseases (2002) 5, 172-179.

8. Krieger JN, Nyberg L Jr, Nickel JC. NIH Consensus Definition and Classification of Prostatitis. JAMA 1999; 282: 236-237.

9. Mené MP, Ginsberg PC, Finkelstein LH, Manfrey SJ, Belkoff L, Ogbolu F. Transurethral microwave hyperthermia in the treatment of chronic nonbacterial prostatitis. J Am Osteopath Assoc. 1997;97(1):25-30.
10. file:///C:/DocumentsandSettings/user/Desktop/ CPPS/NationalGuideline/Chronic pelvic pain_htm

11. Meares EJ. Prostatitis. Med Clin North Am 1991; 75:405-407.

12. Berghuis JP. Psychological and Physiological factors involved in chronic idiopathic prostatitis. J Urol 1998; 159: 1422- 1433.

13. McNaughton W. Chronic prostatitis: a thorough search for etiologically involved microorganisms. Infection 1999;19:119-125.

14. Krieger WW, Jacob J, Ross SO. Detecting urethral and prostatic inflammation in patients with chronic prostatitid. Urology 2000; 55: 186-192.

15. Barbalias GA. Clinical and therapeutic guidelines for chronic prostatitis. Letter to the Editoe. Eur Urol 2000; 37: 116-117.

16. Dimitrakov J. Recent developments in diagnosis and therapy of the prostatitis syndromes. Curr Opinion Urol 2001; 11: 87-91.

17. Moon TD. Questionnare survey of urologists and primary care physicians' diagnostic and treatment practices for prostatitis. Urology 1997; 50: 543544.

18. Cho IR. Prostatic blood flow characteristics in the chronic prostatitis/ chronic pelvic pain syndrome. J Urol 2000; 163: 1130-1133.

\footnotetext{
Abbreviations:

BNI : Bladder neck incision

CPPS : Chronic pelvic pain syndrome

EPS : Expressed prostatic secretion

MIL : Magnetic infrared laser
} 